\title{
Improving the Security of Image Encryption by using Two Chaotic Maps
}

\author{
N. F.Elabady \\ Computer Science \\ Department, Faculty of \\ Computers and Informatics, \\ Benha University, \\ Benha, Egypt
}

\author{
M. I. Moussa \\ Computer Science Department, \\ Faculty of Computers and \\ Informatics, \\ Benha University, \\ Benha, Egypt
}

\author{
S. F. Sabbeh \\ Information System \\ Department, \\ Faculty of Computers and \\ Information, \\ Benha University, Benha, \\ Egypt
}

\author{
H. M.Abdalkader \\ Information System Department, Faculty of Computers and Information, \\ Menofia University, \\ Shebien, Egypt
}

\section{ABSTRACT}

During the recent years several chaotic image encryption algorithms based on one dimensional $(1-D)$ have been proposed, but the drawbacks of small key space, low speed, and low security in 1-D chaotic cryptosystems are obvious. This paper proposes a new image encryption technique based on $1-D$ and $2-D$ chaotic maps which provide more security and big key space. The 1-D chaotic system is induced from a mathematical combination between the logistic map and the cubic chaotic map. It has the advantage of bigger key space and high security analysis such as key space analysis, statistical analysis and sensitivity analysis were carried out. The proposed system uses $1-D$ and $2-D$ chaotic maps for simultaneous encryption of the components $R, G, B$ of the colored image; these three components affect each other. So, the security and confidentiality of image data become more important. The security of digital images and performance speed has become more important since the communications of digital information over network occur more frequently. An image encryption has applications in various fields for example; the internet communication, the multimedia systems, the medical imaging, Tele-medicine and the military communication. The image encryption's aim is to convert the original image to another image that is hard to understand. However, image encryption differs from text encryption due to some intrinsic features of images which include bulk data capacities, high redundancy, strong correlations among pixels, etc. These features make conventional cipher systems such as DES, AES and RSA unsuitable for practical image encryption [1]. The chaos theory is used in cryptography due to its intrinsic features. These properties of chaos includes: sensitivity to initial condition and control parameters, random like behavior and mixing property, etc [2]. Several chaos based image encryption approaches have been described. The first is the confusion approach where the position of pixels scrambled without changing the values of pixels, the application of this approach does not guarantee a good level of security [3]. The second approach is the diffusion which aims to change the value of each pixel in the whole image [4]. Other approach shuffles the positions of plain-image in spatial domain by cat map [5-7]. To enhance the security, the majority of methods propose to mix between more than one approach simultaneously. Some conditions should be fulfilled such as a large key space, randomness of the cipher-image and a high sensitivity on the initial conditions. In [8], an image encryption scheme based on improved 3-D cat map is proposed. Shuffling the positions with Henon map and
\end{abstract}

changing the grey values of image pixels with improved 2-D Logistic map are combined simultaneously to ensure the security of our scheme. New color image encryption algorithms encrypt the three components $R, G, B$ of the color image. The common drawback of these algorithms is: they neglect the correlations between the components $R, G, B$ and are more vulnerable to attack [9-15]. To overcome this problem, a novel color image encryption algorithm based on 1- $D$ chaos map is described for the encryption $R, G$ and $B$ components of color image at the same time and made the three components affect each other [16].

In this paper, a new chaotic map which combines the logistic map and cubic map is used as a $1-D$ chaotic system to shuffle the position of blocks of the three components of image, on the other side the $2-D$ logistic map is used to rearrange the position of image pixels. These two chaotic systems are used to encrypt $R, G$, and $B$ components of color image at the same time and made the three components affect each other. The current algorithm consists of 3 phases. Phase- 1 creates a 1- $D$ chaotic system, its initial values and scrambles the blocks of the image. Phase-2 uses a $2-D$ chaotic system to scrambling the rows and columns and increases the quadratic coupling of the items $y_{i}^{2}, x_{i}^{2}, x_{i} y_{i}$ this provides more security to the system. Phase- 3 uses the 1-D chaotic system again to change the values of pixels.

The rest of this paper is organized as follows: section 2 describes the proposed chaotic system. Section 3 describes the proposed image encryption algorithm.section 4 describes the image decryption algorithm. Section 5 experimental results and performance analysis are reported. Section 6 presents the conclusion.

\section{THE PROPOSED SYSTEM}

The proposed chaotic system uses 1-D formula (1). This formula is written as follows:

$x_{n+1=} \mu x_{n}\left(1-x_{n}\right)\left(2+x_{n}\right)$

When $1.41<\mu<1.59$, the system comes into chaotic state and generate a chaotic sequence in the region $(0,1]$ [17]. The 2-D logistic map formula is written by the following formula:

$$
\begin{aligned}
x_{i+1} & =\mu_{1} x_{i}\left(1-x_{i}\right)+\gamma_{1} y_{i}^{2} \\
y_{i+1} & =\mu_{2} y_{i}\left(1-y_{i}\right)+\gamma_{2}\left(x_{i}^{2}+x_{i} y_{i}\right)
\end{aligned}
$$


Formula (2) increases the quadratic coupling of the items $y_{i}^{2}, x_{i}^{2}, x_{i} y_{i}$ and provides more security to the system. When $2.75<\mu_{1}<3.4,2.7<\mu_{2}<3.45,0.15<\gamma_{1}<0.21$, and $0.13<\gamma_{2}<0.15$, the formula (2) comes into chaotic state and generates a chaotic sequence in the region $(0,1][8]$.

\section{IMAGE ENCRYPTION}

\section{ALGORITHME}

The color image $P$ has the size $M \times N$, where $M$ is the number of row and $N$ is the number of column. Decompose the color image $P$ into three matrixes $R, G$, and $B$. The proposed algorithm generates a total shuffling $P$-box to shuffle the position of blocks of the three components of image, rows and columns the shuffling $P$-box is described as the following:

\section{Shuffling $P$-box ( $W$, formula $(n)$ )}

Step-1. For a given initial values in formula (n) generate the chaotic sequence $S x_{m W}=$ $\left\{x_{1}, x_{2}, \ldots, x_{m}, x_{m+1}, \ldots, x_{m+W}\right\}$ of length $(\mathrm{m}+\mathrm{W})$ by iterating formula for $(m+W)$ times.

Step-2. Delete the first $m$ values of $S x_{m W}$, the chaotic sequence is defined as $S x_{W}=\{$ $\left.x_{m+1}, \ldots, x_{m+W}\right\}$.

Step-3. Sort the sequence $S x_{V U}$ and get the sequence $S x_{W}^{\prime}=\left\{x_{m+1}^{\prime}, x_{m+2}^{\prime}, \ldots, x_{m+W}^{\prime}\right\}$.

Step-4. Determine the position of values $S x_{W}^{\prime}$ in $S x_{W}$ and mark the transform map $T x_{W}=\left\{t_{1}, t_{2}, \ldots, t_{W}\right\}$ where the value $x_{m+i}^{\prime}$ is the same value $x_{t_{i}}$

The proposed algorithm is divided into three phases. The first phase is the Block shuffling phase. The blocks of pixels are shuffled using 1-D chaotic formula (1). The second phase is the Rows and Columns scrambling phase. The pixels in rows and columns are scrambled using the 2-D logistic map formula (2). Finally; the third phase is the Pixels Diffusion phase. The values of pixels are changed using 1-D chaotic system formula (1).

\subsection{Block shuffling}

Each matrices $R, G$, and $B$ of the image $P$ was divided into \# $(V, U)$ of blocks which were combined in $P 1=\left[R_{1}, G_{1}, B_{1}\right]$ , and converted into a one-dimensional vector $V_{P 1}$. The position of these blocks of pixels was shuffled. Phase 1 can be described as follows:

Step-1. Call the shuffling $P$-box(3VU, formula(1)) and get $T x_{V U}$

Step-2. Rearrange the one dimensional vector $V_{P 1}$ according to $T x_{V U}$

Decompose the one dimensional vector $V_{P 1}$ into three $U \times V$ matrices $R_{2}, G_{2}$ and $B_{2}$ then reconstruct the blocks in matrices $R_{2}, G_{2}$, and $B_{2}$ to create $R_{3}, G_{3}$ and $B_{3}$ matrices each of size $M \times N$.

\subsection{Rows and Columns Scrambling}

Determine a chaotic system for the formula (2) and its initial values $\mu_{1}, \mu_{2}, \gamma_{1}, \gamma_{2}, x_{0}$ and $y_{0}$. Rearrange the position of image pixels using the transform maps $T x_{M N}$ and $T y_{M N}$ according to formula (2).

Step-1. Call the shuffling $P$-box(3MV,formula(2)) and get $T x_{M N}, T y_{M N}$

Step-2. Combine the three matrices $R_{3}, G_{3}$ and $B_{3}$ vertically and get matrix $P_{2}$ with $3 M$ rows and $N$ columns.

Step-3. Rearrange the rows of $P_{2}$ according to $T x_{M N}$, which move the $t_{1}$ row to the first row, $t_{2}$ row to the second row, etc. until the rows have been moved and get transformed matrix $P^{\prime}{ }_{2}$.

Step-4. Partition $P^{\prime}{ }_{2}$ into three matrixes $R_{4}, G_{4}$ and $B_{4}$ from top to dawn

Step-5. Combine, $R_{4}, G_{4}$ and $B_{4}$ horizontally and get matrix $P_{3}$ with $M$ rows and $3 N$ columns.

Step-6. Rearrange the columns of $P_{3}$ according to $T y_{M N}$, which move the $t_{1}$ column to the first column, $t_{2}$ column to the second column, etc. until the columns have been moved and get transformed matrix $P_{3}^{\prime}$.

\subsection{Pixels Diffusion}

The values of pixels are changed after the pixel permuted. Given the initial parameters 0 and $x_{0}$ this phase can be described as follows:

Step-1. Use the 1-D chaotic system formula(1) to compute the chaotic sequence

$$
\begin{aligned}
& \text { - } Y M N=\left\{y_{1}, y_{2}, \ldots, y_{3 * M * N}\right\} \\
& \text { - } z_{1 n}=\bmod \left(Y N M^{*} 10^{\wedge} 14,3\right),(n=1,2, \ldots, 3 M \\
& N) \\
& \text { - } z_{2 n}=\bmod \left(Y N M 10^{\wedge} 14,256\right),(n=1,2, \ldots, 3 \\
& M N)
\end{aligned}
$$

Step-2. Partition the transformed matrix $P^{\prime}{ }_{3}$ into three $M \times N$ matrices $R_{5}, G_{5}$ and $B_{5}$ from left to right.

Step-3. Convert the components $R_{5}, G_{5}$ and $B_{5}$ into the vectors $V_{R i}, V_{G i}, V_{B i}$ resp. $(\mathrm{i}=1,2, \ldots, M * N)$.

Step-4. The diffusion formula is $C_{\text {now }}=\left(P_{\text {now }}+z_{2 n}+C_{\text {pre }}+P_{\text {pre }}\right) \bmod 256$ where $C_{\text {now }}$ is the current ciphered value, $P_{\text {now }}$ is the current plain value, $C_{\text {pre }}$ is the previous ciphered value and $P_{p r e}$ is the previous plain value, and the initial values are $C_{0}=0, P_{0}=0$.

Step-5. Repeat

$$
\text { If } z_{1 n}=O \text { then }
$$

Diffuse the current value of $V_{R i}$

If all the values of $V_{R i}$ have changed then

$$
z_{1 n}=1
$$

Diffuse the current value of $V_{G i}$

If all the values of $V_{G i}$ have changed then 


$$
z_{1 n}=2
$$

Diffuse the current value of $V_{B i}$

If all the values of $V_{B i}$ have changed then

$$
z_{1 n}=0
$$
changed

$$
\text { Until all values of } V_{R i}, V_{G i}, V_{B i} \text { is }
$$

Step-6. The resulted ciphered vectors are $V_{R i}^{\prime}, V_{G i}^{\prime}$, $V_{B i}^{\prime}$ where $\left(i=1,2, \ldots, M^{*} N\right)$.

Step-7. The vectors $V_{R i}^{\prime}, V_{G i}^{\prime}, V_{B i}^{\prime}$ are converted into three components $R, G, B$ of the ciphered image $C$.

\section{IMAGE DECRYPTION}

The decryption procedure is similar to that of the encryption process but in the reversed order. The initial parameters and values are as it is in encryption process, the study uses the same methods to obtain $T x_{V U}, T x_{M N}, T y_{M N}, z_{1 n}$, and $z_{2 n}$.

Step-1. Perform the reverse operations to obtain the vectors $V_{R i}, V_{G i}, V_{B i}\left(i=1,2, . ., M^{*} N\right)$ from $\mathrm{C}$. The sequence $z_{1 n}$ is used to remove the effect of diffusion of the ciphered vectors $V_{R i}^{\prime}, V_{G i}^{\prime}, V_{B i}^{\prime}$ according to the following diffusion formula:

$P_{\text {now }}=\left(C_{\text {now }}-z_{2 n}-C_{\text {pre }}-P_{\text {pre }}\right) \bmod 256$

Where $P_{0}=0, C_{0}=0$

Step-2. Perform the reverse operations to remove effect of rows and columns scrambling using the transform maps $T x_{M N}$ and $T y_{M N}$

Step-3. Reverse the operations of the block shuffling phase using the transform maps $T x_{V U}$.

Step-4. Reconstruct the one dimensional $V_{p 1}$ into three $M \times N$ matrices $R_{1}, G_{1}$ and $B_{1}$, then reconstruct the blocks in each matrix $R_{1}, G_{1}$ and $B_{1}$ to create $R, G, B$ matrices of the original colored image $P$.

\section{EXPERIMENTAL RESULTSE}

A The System is implemented by MATLAB R2012a on Windows7 64-bit operating system with an Intel ${ }^{\circledR}$ core $^{\mathrm{TM}}{ }^{\mathrm{M} 5}$ $2430 \mathrm{M}$ CPU $(2.40 \mathrm{GHz})$ and $4 \mathrm{~GB}$ of RAM. For the performance evaluation of the proposed method, 'pepper.jpg' image of size 256x256 have been used. The initial parameters and values

are $\left(\mu=1.57, x_{0}=0.12345678912345, \mu_{1}=3.33, \mu_{2}=3.34\right.$, $\gamma_{1}=0.17, \gamma_{2}=0.14, \mathrm{x}_{10}=0.23456789876543, \mathrm{y}_{10}=0.12345$ $\left.678987654, \mu_{3}=1.56, y_{20}=0.34567891234567\right)$. Figure 1(a) show the original color image.Figures2 (b)-(d) show the $R, G$, $B$ components of the original image. Figures2 (a)-(d) shows the encrypted image and its encrypted components $R, G, B$.

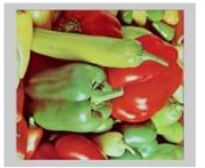

(a)

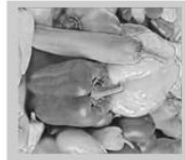

(b)

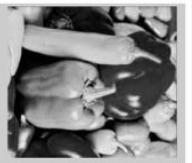

(c)

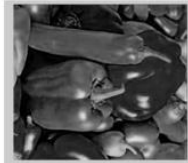

(d)
Fig1. Original image $P$ and its $R, G, B$ components.

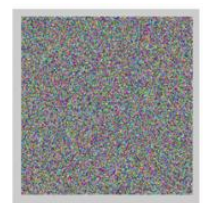

(a)

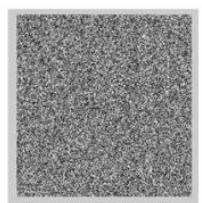

(b)

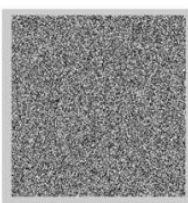

(c)

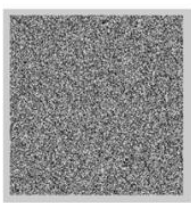

(d)
Fig2. Encrypted image $P$ and its encrypted components $R$, $G$ and $B$.

\subsection{Key Space Analysis}

The key space should be large enough to make brute-force attacks infeasible. The total number of the different keys which can be used in the encryption is defined as the size of the key space. The good encryption algorithm should be sensitive to the secret keys. In current work, the initial conditions and parameters ? $, x_{0}, \mu_{1}, \mu_{2}, \gamma_{1}, \gamma_{2}, x_{10}, y_{10}, \mu_{3}, y_{20}$, are the secret key. If the precision is $10^{\wedge}-14$, the key space size reaches to $10^{\wedge} 140$, it is bigger than $2^{\wedge} 128$. So the key space is large enough to resist the brute-force attacks.

\subsection{Key Sensitivity Analysis}

A good encryption algorithm should be sensitive to the encryption keys in process of both encryption and decryption. When encrypt image, tiny change of keys receive two different cipher images and when decrypt image, if we use wrong key we receive different image. Figures 3(a)-(d) shows the decrypted image of pepper with the correct key $\mu_{3}=1.56$.

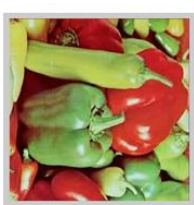

(a)

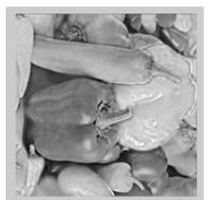

(b)

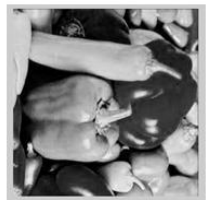

(c)

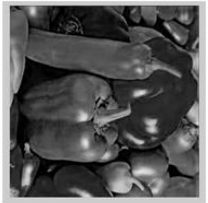

(d)
Fig3. Decrypted image and its components with correct parameters and its components.

Figure 4(a)-(d) shows the decrypted image of pepper_with a wrong encryption key $\mu_{3}=1.56000000000001$. So it can be concluded that the algorithm is sensitive to the key, a small change of the key will generate a completely different decryption result and cannot get the correct plain-image.

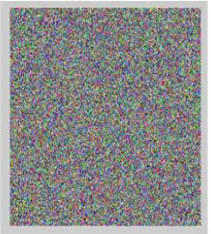

(a)

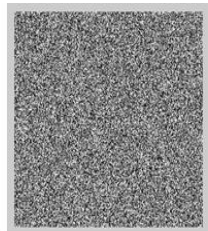

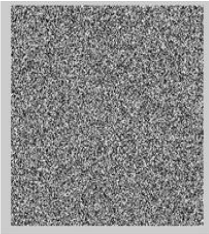

(c)

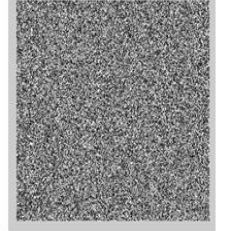

(d)
Fig4. Decrypted image with and its components incorrect parameters

\subsection{Correlation Analysis}

Randomly, select 3000 pairs of adjacent pixels in three directions (vertical, horizontal and diagonal) to test the correlation among pixels, and plot the corresponding distributions. The correction coefficient is calculated for each pair using the following formulas: 
$r_{x y}=\frac{\operatorname{cov}(x, y)}{\sqrt{D(x)} \sqrt{D(y)}}$

$$
\operatorname{cov}(x, y)=\frac{1}{N} \sum_{i=1}^{N}\left(x_{i}-E(x)\right)\left(y_{i}-E(y)\right)
$$

$$
\begin{aligned}
& E(x)=\frac{1}{N} \sum_{i=1}^{N}\left(x_{i}\right) \\
& E(y)=\frac{1}{N} \sum_{i=1}^{N}\left(y_{i}\right) \\
& D(x)=\sum_{i=1}^{N}\left(x_{i}-E(x)\right)^{2} \\
& D(y)=\sum_{i=1}^{N}\left(y_{i}-E(y)\right)^{2}
\end{aligned}
$$

Where $x, y$ are gray value of two adjacent pixels. The vertical and diagonal directions results are obtained, they are shown in Table 1. The correlation of two adjacent pixels in plain image closed to 1 , while in ciphered image it closed to 0 which demonstrate that the image encryption could effectively resist statistical attack.

Table 1: The correlation test of the components $R, G, B$ of the plain image and the encrypted image.

\begin{tabular}{|c|c|c|c|}
\hline Image & Component & $\begin{array}{c}\text { Plain } \\
\text { image }\end{array}$ & $\begin{array}{c}\text { Encrypted } \\
\text { image }\end{array}$ \\
\hline \multirow{2}{*}{ pepper } & $\mathrm{R}$ & 0.9602 & 0.0014 \\
& $\mathrm{G}$ & 0.9790 & 0.0009 \\
\cline { 2 - 4 } & $\mathrm{B}$ & 0.9557 & 0.00013 \\
\cline { 2 - 4 } & &
\end{tabular}

\subsection{Information Entropy}

The information entropy is defined to express the degree of uncertainties in the system [18].Using it to express uncertainties of the image information. The information entropy measures the distribution of gray value in image. If the uncertainty of image is greater, the entropy is bigger and the decrypting process of the image requires more information too. On the contrary, the more orderly of the encrypted image is the smaller of the information entropy. The information entropy equation is written as follows:

$H(m)=\sum_{i=0}^{M} P\left(m_{i}\right) \log _{2} 1 \frac{1}{P\left(m_{i}\right)}$

Where $M$ is the total number of symbols $m_{i}, P\left(m_{i}\right)$ represents the probability of occurrence of symbol $m_{i}$ and the logarithm bases 2 so that the entropy is expressed in bits. The information entropy of an ideal random image is 8 . Table 2 shows that the entropy of encryption image is very close to 8 . This means that the encrypted images are close to a random source and the proposed algorithm is secure against the entropy attack.

Table2. Information entropy of original image and decrypted

\begin{tabular}{|c|c|c|c|}
\hline Image & Component & Plain image & $\begin{array}{c}\text { Encrypted } \\
\text { image }\end{array}$ \\
\hline \multirow{2}{*}{ pepper } & $\mathrm{R}$ & 7.3921 & 7.9973 \\
& $\mathrm{G}$ & 7.6813 & 7.9973 \\
\cline { 2 - 4 } & $\mathrm{B}$ & 7.1756 & 7.9966 \\
\cline { 2 - 4 } & & & \\
\hline
\end{tabular}

\subsection{Histogram analysis}

The image histogram illustrated how pixels in an image are distributed by graphing the number of pixels at each color intensity level. Figure 5(a)-(c) shows the histogram of plain components $R, G, B$.

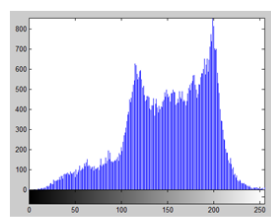

(a)

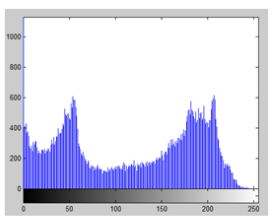

(b)

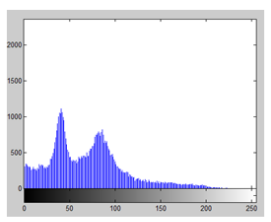

(c)
Fig5. The histogram of original image's components $R, G$, and $B$. (a) The histogram of $R$ component. (b) The histogram of $G$ component. (c) The histogram of $B$ component.

Figures 6(a)-(c) shows the histogram of encrypted components R, G, and B. The histogram of the cipher image is fairly uniform and significantly different from that of the original image

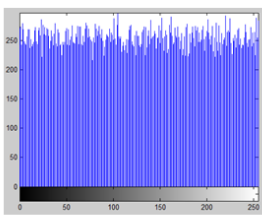

(a)

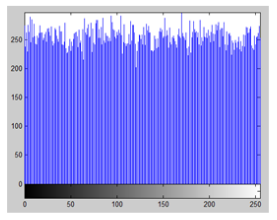

(b)

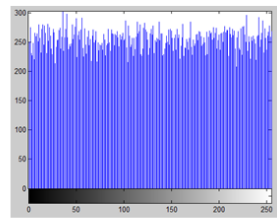

(c)
Fig 6: The histogram of the encrypted image's components $R, G$, and $B$. (a) The histogram of encrypted component $R$.

(b) The histogram of encrypted component $G(c)$ The histogram of encrypted component $B$.

\subsection{NPCR and UACI Analysis}

The measures NPCR and UACI test the different range between two images. NPCR stands for the changing rate of the number of pixels while one pixel of plain image is changed. UACI stands for the average intensity of the differences between the plain image and the cipher image. Calculate $\operatorname{NPCR}_{\mathrm{R}, \mathrm{G}, \mathrm{B}}$ and $\mathrm{UACI}_{\mathrm{R}, \mathrm{G}, \mathrm{B}}$ by using the following formulas:

$N P C R_{R, G, B}=\frac{\sum_{i j} D_{R, G, B}(i, j)}{W \times H} \times 100 \%$

$U A C I_{R, G, B}=\frac{1}{W \times H}\left[\frac{\sum_{i j}\left|C_{R, G, B}(i, j)-C^{\prime}{ }_{R, G, B}(i, j)\right|}{255}\right] \times 100 \%$

Where $\mathrm{W}$ and $\mathrm{H}$ are the width and height of the plain image, $C_{R, G, B}(i, j)$ and $C_{R, G, B}^{\prime}(i, j)$ are the $(i, j)$ pixel values of the encrypted image before and after changing the value of one pixel the pixel $(i, j)$ of the plain image respectively. The value $D_{R, G, B}(i, j)$ equals zero if $C_{R, G, B}(i, j)=C_{R, G, B}^{\prime}(i, j)$, otherwise $D_{R, G, B}(i, j)$ equal one. In Table $3, \operatorname{NPCR}_{R, G, B}$ is over $99 \%$ and $\mathrm{UACI}_{R, \mathrm{G}, \mathrm{B}}$ is over $33 \%$. These results show that, the proposed algorithm is very sensitive to tiny changes in the plain image, even if there is a change in only one pixel, the decrypted images is completely different. Thus, the algorithm is robust against differential attack. 
Table3. NPCR and UACI results

\begin{tabular}{|l|l|l|l|l|}
\hline \multirow{2}{*}{ Image } & \multirow{3}{*}{} & \multicolumn{3}{|c|}{ Proposed algorithm } \\
\cline { 3 - 5 } & & $\mathbf{R}$ & G & B \\
\hline Pepper & $\begin{array}{l}\text { NPCR } \\
\%\end{array}$ & 99.6674 & 99.6338 & 99.5712 \\
\cline { 2 - 5 } & $\begin{array}{l}\text { UACI } \\
\%\end{array}$ & 33.6665 & 33.5003 & 33.4723 \\
\hline
\end{tabular}

\subsection{PSNR analysis}

The Peak Signal-to-Noise Ratio (PSNR) reflects the encryption quality. The Mean Square Error (MSE) is the cumulative squared error between the original and the decrypted image. The lower value of MSE means lesser error.

$$
\begin{aligned}
& \operatorname{MSE}_{R, G, B}=\sum_{i} \sum_{j} \frac{P_{R, G, B}(i, j)-C_{R, G, B}(i, j)}{M x N} \\
& \operatorname{PSNR}_{R, G, B}=20 * \log 10\left(\frac{255}{\operatorname{sgrt}\left(M S E_{R, G, B}\right)}\right)
\end{aligned}
$$

Where $P_{R, G, B}$ the original is image and $C_{R, G, B}$ is the encrypted image. The lower value of PSNR is the better encryption quality. The results are showed in table 4.

\section{Table4. PSNR results}

\begin{tabular}{|c|c|c|c|}
\hline \multirow{2}{*}{ Image } & \multicolumn{3}{|c|}{ Proposed algorithm } \\
\cline { 2 - 4 } & $\mathrm{R}$ & $\mathrm{G}$ & $\mathrm{B}$ \\
\hline Pepper & 9.0945 & 7.7804 & 7.7578 \\
\hline
\end{tabular}

\subsection{Randomness test}

The security of cryptosystem has some properties such as good distribution, long period, high complexity and efficiency. In particular, the outputs of the cryptosystem must be unpredictable in the absence of knowledge of the inputs. Recently, the NIST designed a set of different statistical tests to test randomness of binary sequences produced by either hardware or software based cryptographic random or pseudorandom number generators [19]. These tests focus on a variety of different types of non-randomness that could exist in a sequence. We use the NIST tests suite in order to test the randomness of the proposed algorithm. In all tests if the computed P-value is less than 0.01 , the sequence is nonrandom. Otherwise, the sequence is random. Table 5 depicts the NIST tests results and illustrates that the image sequences encrypted by the proposed system pass all the statistical tests with high P-values.

Table5. NIST Randomness Tests

\begin{tabular}{|l|l|l|}
\hline Image & Test Name & P-value \\
\hline \multirow{4}{*}{} & Approximate Entropy & 0.817929 \\
\cline { 2 - 3 } & Frequency & \\
\cline { 2 - 3 }$\underset{\overbrace{}}{\mathbb{\overbrace}}$ & Block Frequency & 0.079125 \\
\cline { 2 - 3 } & Cumulative sums & 0.968310 \\
\cline { 2 - 3 } & FFT & 0.149466 \\
\cline { 2 - 3 } & Linear complexity & 0.257962 \\
\cline { 2 - 3 } & Run & 0.828726 \\
\hline
\end{tabular}

\begin{tabular}{|l|l|l|}
\hline \multirow{4}{*}{} & Longest Run & 0.166992 \\
\cline { 2 - 3 } & Overlapping template & 0.137277 \\
\cline { 2 - 3 } & Rank & 0.611852 \\
\cline { 2 - 3 } & Serial P-vaue1 & 0.672378 \\
\cline { 2 - 3 } & Serial P-value2 & 0.640758 \\
\cline { 2 - 3 } & Universal & 0.814821 \\
\hline
\end{tabular}

\section{CONCLUSION}

In this paper, the security of the system is increased by using two chaotic systems. The proposed system consisted of three phases. In the first phase a new $1-D$ chaotic map is used to scramble the blocks of image. In the second phase a 2-D logistic map is used to shuffle the position in rows and columns. Finally the 1- $D$ chaotic map is used again to change the values of the pixels. The simulation results show that the correlation coefficient between two adjacent pixels in the cipher image is far less than that of plain image; this indicates that the cipher image has a high performance of resisting statistical attack and high sensitive to security key. The key space of the proposed system is about $10^{\wedge} 140$, it is bigger than the key space of using only a $1-D$ chaotic map. The keys space is large enough to resist brute-force attack. 3D logistic map will be used in future work to increase the security of algorithm.

\section{REFERENCE}

[1] S. Li, G. Chen, A. Cheung, B. Bhargava, K.-T. Lo, On the Design of Perceptual MPEG video Encryption Algorithms, CoRR abs/cs/0501014, 2005.Encryption Algorithms, CoRR abs/cs/0501014, 2005.

[2] C. Fu, Z. Zhang and Y. Cao, "An Improved Image Encryption Algorithm Based on Chaotic Maps," Third In-ternational Conference on Natural Computation, Vol. 3, Washington, 2007, pp. 24-27.

[3] S. Li, C. Li, G. Chen, N.G. Bourbakis, K.T. Lo, "A general quantitative cryptanalysis of permutation-only multimedia ciphers against plaintext attacks", Signal Processing: Image Communication 23 (3) (2008) 212 223

[4] Xin Zhang, Webin Chen,"Anew Chaotic Algorithm For Image Encryption", pp.889-892 IEEE ICALIP 2008.

[5] G.R. Chen, Y.B. Mao, and Charles K. Chui, "A symmetric image encryption scheme based on 3D chaotic cat maps", Chaos, Solitons \& Fractals, 2004, pp. 749-741.

[6] Y.N.SI, B.S KANG, "Digital Image Scrambling Based on Improved Arnold Transformation", Chinese Comp. Technology and development, 2008, pp.74-79.

[7] Y. Wang, Z.W. Zhao, and L.L. Zou, "A Fault-tolerable Encryption Algorithm for Two-dimensional Digital Image", 2007 2nd IEEE Conference on Industrial Electronics and Applications, 2007, pp. 2737-2741.

[8] Hongjuan Liu, Zhiliang Zhu, Huiyan Jiang, Beilei Wang. "A Novel Image EncryptionAlgorithm Based on Improved 3D Chaotic Cat Map", The 9th International Conference for Young Computer Scientists, 2008.

[9] K.Sakthidasan Sankaran and B.V.Santhosh Krishna," A New Chaotic Algorithm for Image Encryption and Decryption of Digital Color Images", International 
Journal of Information and Education Technology, Vol. 1, No. 2, June 2011.

[10] LIU Xiangdong, Zhang Junxing, Zhang Jinhai, He Xiqin,"Image Scrambling Algorithm Based on Chaos Theory and Sorting Transformation".IJCSNS International Journal of Computer Science and Network Security, VOL.8 No.1, January 2008.

[11] Qais H. Alsafasfeh, Aouda A. Arfoa," Image Encryption Based on the General Approach for Multiple Chaotic Systems", Journal of Signal and Information Processing, 2011, 2, 238-244.

[12] H. Zhang, X.F. Wang, Z.H. Li, and D.H. Liu, "A Fast Image Encryption Algorithm Based on Chaos System and Henon Map", Chinese Journal of Computer Research and Development,2005,pp.2137-2142.

[13] X.J. Li, J.H. Peng, and N. Xu, et al. "Image Encryption Algorithm Based on 2D Hyper chaotic Sequences", Journal of Image and Graphics, 2003, pp. 1172-1177.

[14] Sudhir Keshari, 2Dr. S. G. Modani," Image Encryption Algorithm based on Chaotic Map Lattice and Arnold cat map for Secure Transmission", IJCST Vol. 2, Issue 1, March 2011.
[15] Guoji Zhang a, Qing Liu b,," A novel image encryption method based on total shuffling scheme", Optics Communications 284 (2011) 2775-2780.

[16] Xingyuan Wangn, LinTeng,XueQin," A novel colour image encryption algorithm based on chaos", Signal Processing 92 (2012) 1101-1108.

[17] N. F.Elabady , H. M.Abdalkader , M. I. Moussa and S. F. Sabbeh, "Image Encryption Based on New-One dimensional chaotic Map", Second International Conference on Engineering and Technology ,GUC,Egypt,2013.

[18] Wang Y, Wong K-W, Liao X, Chen G,"A new chaosbased fast image encryption algorithm", Applied Soft Computing, Volume 11 Issue 1, January, 2011

[19] Rukhin, A. et al. (2010b). A Statistical Test Suite for Random and Pseudorandom Number Generators for Cryptographic Applications. NIST Special Publication 800-22, Revision 1a. 